\title{
SOLVABILITY OF EQUATIONS BY QUADRATURES AND NEWTON'S THEOREM
}

\author{
AsKold KhovanskiI \\ Department of Mathematics, University of Toronto, Toronto, Canada \\ dedicated to Victor Buchstaber's 75th birthday
}

\begin{abstract}
Picard-Vessiot theorem (1910) provides a necessary and sufficient condition for solvability of linear differential equations of order $n$ by quadratures in terms of its Galois group. It is based on the differential Galois theory and is rather involved. J.Liouville in 1839 found an elementary criterium for such solvability for $n=2$. J.F.Ritt simplified Liouville's theorem (1948). In 1973 M. Rosenlicht proved a similar criterium for arbitrary $n$. Rosenlicht work relies on the valuation theory and is not elementary. In these notes we show that the elementary Liouville-Ritt method based on developing solutions in Puiseux series as functions of a parameter works smoothly for arbitrary $n$ and proves the same criterium.
\end{abstract}

\section{INTRODUCTION}

Consider a homogeneous linear differential equation

$$
y^{(n)}+a_{1} y^{(n-1)}+\cdots+a_{n} y=0
$$

whose coefficients $a_{i}$ belong to a differential field $K$.

Theorem 1. If the equation (1) has a non zero solution representable by generalized quadratures over $K$ then it necessarily has a solution of the form $y_{1}=\exp z$ where $z^{\prime}$ is algebraic over $K .^{1}$

The following lemma is obvious.

Lemma 2. Assume that the equation (1) has a non zero solution $y_{1}$ representable by generalized quadratures over $K$. Then the equation (1) can be solved by generalized quadratures over $K$ if and only if the linear differential equation of order $(n-1)$ over the differential field $K\left(y_{1}\right)$ obtained from (1) by the reduction of order using the solution $y_{1}$ is solvable by generalized quadratures over $K\left(y_{1}\right)$.

Indeed on one hand each solution of the equation obtained from (1) by the reduction of order using $y_{1}$ can be expressed in the form $\left(y / y_{1}\right)^{\prime}$ where $y$ is a solution of (1). On the other hand any solution $y$ of the equation $\left(y / y_{1}\right)^{\prime}=u$, where $u$ is

Key words and phrases. linear differential equation, solvability by quadratures.

The work was partially supported by the Canadian Grant No. 156833-17.

${ }^{1}$ In the section 5 we provide a generalization of Theorem 1 for nonlinear homogeneous equations. 
represented by generalized quadratures over $K\left(y_{1}\right)$, is representable by generalized quadratures over $K$, assuming that $y_{1}$ representable by generalized quadratures over $K$.

Thus Theorem 1 provides the following criterium for solvability of the equation (1) by generalized quadratures.

Theorem 3. The equation (1) is solvable by generalized quadratures over $K$ if and only if the following conditions hold:

1) the equation (1) has a solution $y_{1}$ of the form $y_{1}=\exp z$ where $z^{\prime}=f$ is algebraic over $K$,

2) the linear differential equation of order $(n-1)$ over $K\left(y_{1}\right)$ obtained from (1) by the reduction of order using the solution $y_{1}$ is solvable by generalized quadratures over $K\left(y_{1}\right)$.

The standard proof (E. Picard and E. Vessiot, 1910) of Theorem 1 uses the differential Galois theory and is rather involved (see [4]). ${ }^{2}$

In the case when the equation (1) is a Fuchsian differential equation and $K$ is the field of rational function of one complex variable Theorem 3 has a topological explanation (see [3] which allows to prove much stronger version of this result). But in general case Theorem 3 does not have a similar visual explanation.

In these notes I discuss an elementary proof of Theorem 1 based on old arguments suggested by J. Liouville, J.F.Ritt and M. Rosenlicht.

Maxwell Rosenlicht in 1973 proved [2] the following theorem.

Theorem 4. Let $n$ be a positive integer, and let $Q$ be a polynomial in several variables with coefficients in a differential field $K$ and of total degree less than $n$. Then if the equation

$$
u^{n}=Q\left(u, u^{\prime}, u^{\prime \prime}, \ldots\right)
$$

has a solution representable by generalized quadratures over $K$, it has a solution algebraic over $K$.

The logarithmic derivative $u=y^{\prime} / y$ of any solution of the equation (1) satisfies the associated with (1) generalized Riccati's equation of order $(n-1)$, which is a particular case of the equation (2). Rosenlicht showed that Theorem 1 easily follows from Theorem 4 applied to the corresponding generalized Riccati's equation (see section 5). In modern differential algebra abstract fields equipped with an operation of differentiation are considered. The Rosenlicht's proof of Theorem 4 is not elementary: it is applicable to abstract differential fields of characteristic zero and makes use of the valuation theory. ${ }^{3}$.

J. Liouvillive and J.F.Ritt deal with fields of meromorphic functions with the usual differentiation. This point of view is natural for the analytic theory of differential equations. Solutions of differential equations are function, not elements

\footnotetext{
${ }^{2} \mathrm{~A}$ generaliztion of Theorem 1 for nonlinear homogeneous equations presented in the section 5 does not follow from the Picard-Vessio theory.

${ }^{3}$ According to Michael Singer the valuation theory used in Rosenlicht's proof is a fancy way of using power series methods (private communication).
} 
of abstract differential fields. Analytic function could be multivalued, could have singularities and so on. For applications of results from differential algebra to the theory of differential equations some extra work is needed. In these notes we deal with functional differential fields. We present needed material in sections $3-6$, mainly following the presentation from the book [3].

Joseph Liouville in 1839 proved Theorem 1 for $n=2$. Joseph Fels Ritt in 1948 simplified his proof (see [1]). The logarithmic derivative $u=y^{\prime} / y$ of any solution $y$ of the homogeneous linear differential equation (1) of second order satisfies the Riccati's equation

$$
u^{\prime}+a_{1} u+a_{2}+u^{2}=0
$$

To prove Theorem 1 for $n=2$ J.Liouville and J.F.Ritt proved first Theorem 4 for the Riccati's equation (3). To do that J.F.Ritt considered a special one parametric family of solutions of (3) and used an expansion of these solutions as functions of the parameter into converging Puiseux series. J.F.Ritt used a generalization of the following theorem based on ideas suggested by Newton.

Consider an algebraic function $z(y)$ defined by an equation $P(y, z)=0$ where $P$ is a polynomial with coefficients in a subfield $K$ of $\mathbb{C}$. Then all branches of the algebraic function $z(y)$ at the point $y=\infty$ can be developed into converging Puiseux series whose coefficients belong to a finite extension of the field $K$.

A generalized Newton's Theorem claims that the similar result holds if instead of a numerical field of coefficient one takes a field $K$ whose element are meromorphic functions on a connected Riemann surface. In the J.F.Ritt's book [1] this result is proved in the same way as its classical version using the Newton's polygon method.

Unfortunately J.F.Ritt's proof is written in old mathematical language and does not fit into our presentation. Theorem 13 provides an exact statement of the generalized Newton's Theorem. It is presented without proof: main arguments proving it are well known and classical. One also can obtain a proof modifying J.F.Ritt's exposition. Theorem 13 plays a crucial role in these notes. For the sake of completeness I will present its modern proof in a separate paper.

In these notes I discuss a proof of Theorem 4 which does not rely on the valuation theory. It generalizes J.F.Ritt's arguments (makes use of the Puiseux expansion via a generalized Newton's Theorem) and provides an elementary proof of the classical Theorem 1.

The idea of the proof goes back to Liouville and J.F.Ritt. I came up with it trying to understand and comment the classical book written by J.F.Ritt [1].

I am grateful to Michael Singer who invited me to write comments for a new edition of this book.

\section{Generalized QUADRATURES OVER FUnCTIONAL DIFFERENTIAL FIELDS}

In the sections 1-4 we present definitions and general statements related to functional and abstact differential fields and classes of their extensions including extensions by generalized quadratures. We follow mainly the presentation from the book [3]. In the section 5 we define the generalized Riccati's equation and reduce Theorem 1 to Theorem 4 . 
1. Abstract differential fields. A field $F$ is said to be a differential field if an additive map $a \rightarrow a^{\prime}$ is fixed that satisfies the Leibnitz rule $(a b)^{\prime}=a^{\prime} b+a b^{\prime}$. The element $a^{\prime}$ is called the derivative of $a$. An element $y \in F$ is called $a$ constant if $y^{\prime}=0$. All constants in $F$ form the field of constants. We add to the definition of differential field an extra condition that the field of constants is the field of complex numbers (for our purpose it is enough to consider fields satisfying this condition). An element $y \in F$ is said to be: an exponential of integral of $a$ if $y^{\prime}=a y$; an integral of $a$ if $y^{\prime}=a$. In each of these cases, $y$ is defined only up to a multiplicative or an additive complex constant.

Let $K \subset F$ be a differential subfield in $F$. An element $y$ is said to be an integral over $K$ if $y^{\prime}=a \in K$. An exponential of integral over $K$ is defined similarly.

Suppose that a differential field $K$ and a set $M$ lie in some differential field $F$. The adjunction of the set $M$ to the differential field $K$ is the minimal differential field $K\langle M\rangle$ containing both the field $K$ and the set $M$. We will refer to the transition from $K$ to $K\langle M\rangle$ as adjoining the set $M$ to the field $K$.

Definition 1. An extension $F$ of a differential field $K$ is said to be:

1) a generalized extension by integral if there are $y \in F$ and $f \in K$ such that $y^{\prime}=f, y$ is transcendental over $K$, and $F$ is a finite extension of the field $K\langle y\rangle$;

2) a generalized extension by exponential of integral if there are $y \in F, f \in K$ such that $y^{\prime}=f y, y$ is transcendental over $K$, and $F$ is a finite extension of the field $K\langle y\rangle$;

3) an extension by generalized quadratures if there exists a chain of differential fields $K=F_{0} \subset \cdots \subset F_{n} \supset F$ such that $F_{i+1}=F_{i}<y_{i}>$ for every $i=0, \ldots$, $n-1$ where $y_{i}$ is an exponential of integral, an integral, or an algebraic element over the differential field $F_{i}$.

An element $a \in F$ is representable by generalized quadratures over $K, K \subset F$, if it is contained in a certain extension of the field $K$ by generalized quadratures. The following lemma is obvious.

Lemma 5. An extension $K \subset F$ is an extension by generalized quadratures if there is a chain $K=F_{0} \subset \cdots \subset F_{n}$ such that $F \subset F_{n}$ and for every $i=0, \ldots, n-1$ or $F_{i+1}$ is a finite extension of $F_{i}$, or $F_{i+1}$ is a generalized extension by integral of $F_{i}$, or $F_{i+1}$ is a generalized extension by exponential integral of $F_{i}$.

2. Functional differential fields and their extensions. Let $K$ be a subfield in the field $F$ of all meromorphic functions on a connected domain $U$ of the Riemann sphere $\mathbb{C}^{1} \cup \infty$ with the fixed coordinate function $x$ on $\mathbb{C}^{1}$. Suppose that $K$ contains all complex constants and is stable under differentiation (i.e. if $f \in K$, then $f^{\prime}=$ $d f / d x \in K)$. Then $K$ provides an example of a functional differential field.

Let us now give a general definition.

Definition 2. Let $U, x$ be a pair consisting of a connected Riemann surface $U$ and a non constant meromorphic function $x$ on $U$. The map $f \rightarrow d f / d x$ defines the derivation in the field $F$ of all meromorphic functions on $U$ (the ratio of two meromorphic 1-forms is a well-defined meromorphic function). A functional differential field $K$ is any differential subfield of $F$ (containing all complex constants).

With any function $f$ from a functional differential field $K$ let us associate its meromorphic germ $f_{a}$ at point $a \in U$. The differential field $K$ is isomorphic to the differential field $K_{a}$ of germs at a of function belonging to $K$. 
The following construction helps to extend functional differential fields. Let $K$ be a differential subfield of the field of meromorphic functions on a connected Riemann surface $U$ equipped with a meromorphic function $x$. Consider any connected Riemann surface $V$ together with a nonconstant analytic map $\pi: V \rightarrow U$. Fix the function $\pi^{*} x$ on $V$. The differential field $F$ of all meromorphic functions on $V$ with the differentiation $\varphi^{\prime}=d \varphi / \pi^{*} d x$ contains the differential subfield $\pi^{*} K$ consisting of functions of the form $\pi^{*} f$, where $f \in K$. The differential field $\pi^{*} K$ is isomorphic to the differential field $K$, and it lies in the differential field $F$. For a suitable choice of the surface $V$, an extension of the field $\pi^{*} K$, which is isomorphic to $K$, can be done within the field $F$.

Suppose that we need to extend the field $K$, say, by an integral $y$ of some function $f \in K$. This can be done in the following way. Consider the covering of the Riemann surface $U$ by the Riemann surface $V$ of an indefinite integral $y$ of the form $f d x$ on the surfave $U$. By the very definition of the Riemann surface $V$, there exists a natural projection $\pi: V \rightarrow U$, and the function $y$ is a single-valued meromorphic function on the surface $V$. The differential field $F$ of meromorphic functions on $V$ with the differentiation $\varphi^{\prime}=d \varphi / \pi^{*} d x$ contains the element $y$ as well as the field $\pi^{*} K$ isomorphic to $K$. That is why the extension $\pi^{*} K\langle y\rangle$ is well defined as a subfield of the differential field $F$. We mean this particular construction of the extension whenever we talk about extensions of functional differential fields. The same construction allows to adjoin a logarithm, an exponential, an integral or an exponential of integral of any function $f$ from a functional differential field $K$ to $K$. Similarly, for any functions $f_{1}, \ldots, f_{n} \in K$, one can adjoin a solution $y$ of an algebraic equation $y^{n}+f_{1} y^{n-1}+\cdots+f_{n}=0$ or all the solutions $y_{1}, \ldots, y_{n}$ of this equation to $K$ (the adjunction of all the solutions $y_{1}, \ldots, y_{n}$ can be implemented on the Riemann surface of the vector-function $\left.\mathbf{y}=y_{1}, \ldots, y_{n}\right)$. In the same way, for any functions $f_{1}, \ldots, f_{n+1} \in K$, one can adjoin the $n$-dimensional $\mathbb{C}$-affine space of all solutions of the linear differential equation $y^{(n)}+f_{1} y^{(n-1)}+\cdots+f_{n} y+f_{n+1}=0$ to $K$. (Recall that a germ of any solution of this linear differential equation admits an analytic continuation along a path on the surface $U$ not passing through the poles of the functions $f_{1}, \ldots, f_{n+1}$.)

Thus, all above-mentioned extensions of functional differential fields can be implemented without leaving the class of functional differential fields. When talking about extensions of functional differential fields, we always mean this particular procedure.

The differential field of all complex constants and the differential field of all rational functions of one variable can be regarded as differential fields of functions defined on the Riemann sphere.

3. Classes of functions and operations on multivalued functions. An indefinite integral of an elementary function is a function rather than an element of an abstract differential field. In functional spaces, for example, apart from differentiation and algebraic operations, an absolutely non-algebraic operation is defined, namely, the composition. Anyway, functional spaces provide more means for writing "explicit formulas" than abstract differential fields. Besides, we should take into account that functions can be multivalued, can have singularities and so on.

In functional spaces, it is not hard to formalize the problem of unsolvability of equations in explicit form. One can proceed as follows: fix a class of functions and say that an equation is solvable explicitly if its solution belongs to this class. 
Different classes of functions correspond to different notions of solvability.

3.1. Defining classes of functions by the lists of data. A class of functions can be introduced by specifying a list of basic functions and a list of admissible operations. Given the two lists, the class of functions is defined as the set of all functions that can be obtained from the basic functions by repeated application of admissible operations. Below, we define the class of functions representable by generalized quadratures and the class of functions representable by generalized quadratures over a functional differential field $K$ in exactly this way.

Classes of functions, which appear in the problems of solvability of differential equations by quadratures, contain multivalued functions. Thus the basic terminology should be made clear. We work with multivalued functions "globally", which leads to a more general understanding of classes of functions defined by lists of basic functions and of admissible operations. A multivalued function is regarded as a single entity. Operations on multivalued functions can be defined. The result of such an operation is a set of multivalued functions; every element of this set is called a function obtained from the given functions by the given operation. A class of functions is defined as the set of all (multivalued) functions that can be obtained from the basic functions by repeated application of admissible operations.

3.2. Operations on multivalued functions. Let us define, for example, the sum of two multivalued functions on a connected Riemann surface $U$.

Definition 3. Take an arbitrary point $a$ in $U$, any germ $f_{a}$ of an analytic function $f$ at the point $a$ and any germ $g_{a}$ of an analytic function $g$ at the same point $a$. We say that the multivalued function $\varphi$ on $U$ generated by the germ $\varphi_{a}=f_{a}+g_{a}$ is representable as the sum of the functions $f$ and $g$.

For example, it is easy to see that exactly two functions of one variable are representable in the form $\sqrt{x}+\sqrt{x}$, namely, $f_{1}=2 \sqrt{x}$ and $f_{2} \equiv 0$. Other operations on multivalued functions are defined in exactly the same way. For a class of multivalued functions, being stable under addition means that, together with any pair of its functions, this class contains all functions representable as their sum. The same applies to all other operations on multivalued functions understood in the same sense as above.

In the definition given above, not only the operation of addition plays a key role but also the operation of analytic continuation hidden in the notion of multivalued function. Indeed, consider the following example. Let $f_{1}$ be an analytic function defined on an open subset $V$ of the complex line $\mathbb{C}^{1}$ and admitting no analytic continuation outside of $V$, and let $f_{2}$ be an analytic function on $V$ given by the formula $f_{2}=-f_{1}$. According to our definition, the zero function is representable in the form $f_{1}+f_{2}$ on the entire complex line. By the commonly accepted viewpoint, the equality $f_{1}+f_{2}=0$ holds inside the region $V$ but not outside.

Working with multivalued functions globally, we do not insist on the existence of a common region, were all necessary operations would be performed on singlevalued branches of multivalued functions. A first operation can be performed in a first region, then a second operation can be performed in a second, different region on analytic continuations of functions obtained on the first step. In essence, this more general understanding of operations is equivalent to including analytic continuation to the list of admissible operations on the analytic germs. 
4. Functions representable by generalized quadratures. In this section we define functions of one complex variable representable by generalized quadratures and functions representable by generalized quadratures over a functional differential field. We also discuss a relation of these notions with extensions of functional differential fields by generalized quadratures. First we'll present needed lists of basic functions and of admissible operations.

\section{List of basic elementary functions.}

1. All complex constants and an independent variable $x$.

2. The exponential, the logarithm, and the power $x^{\alpha}$ where $\alpha$ is any constant.

3. The trigonometric functions sine, cosine, tangent, cotangent.

4. The inverse trigonometric functions arcsine, arccosine, arctangent, arccotangent.

Lemma 6. Basic elementary functions can be expressed through the exponentials and the logarithms with the help of complex constants, arithmetic operations and compositions.

Lemma 6 can be considered as a simple exercise. Its proof can be found in [3].

\section{List of some classical operations.}

1) Operation of composition takes functions $f, g$ to the function $f \circ g$.

2) The arithmetic operations take functions $f, g$ to the functions $f+g, f-g$, $f g$, and $f / g$.

3) The operation of differentiation takes function $f$ to the function $f^{\prime}$.

4) The operation of integration takes function $f$ to a solution of equation $y^{\prime}=f$ (the function $y$ is defined up to an additive constant).

5) The operation of taking exponential of integral takes function $f$ to a solution of equation $y^{\prime}=f y$ (the function $y$ is defined up to a multiplicative constant).

6) The operation of solving algebraic equations takes functions $f_{1}, \ldots, f_{n}$ to the function $y$ such that $y^{n}+f_{1} y^{n-1}+\cdots+f_{n}=0$ ( the function $y$ is not quite uniquely determined by functions $f_{1}, \ldots, f_{n}$ since an algebraic equation of degree $n$ can have $n$ solutions).

Definition 4. The class of functions of one complex variable representable by generalized quadratures is defined by the following data:

List of basic functions: basic elementary functions.

List of admissible operations: Compositions, Arithmetic operations, Differentiation, Integration,Operation of taking exponential of integral, Operation of solving algebraic equations.

Theorem 7. A (possibly multivalued) function of one complex variable belongs to the class of functions representable by generalized quadratures if and only if it belongs to some extension of the differential field of all constant functions of one variable by generalized quadratures.

Theorem 7 follows from Lemma 6 (All needed arguments can be found in [3]).

Let $K$ be a functional differential field consisting of meromorphic functional on a connected Riemann surface $U$ equipped with a meromorphic function $x$.

Definition 5. The class of functions representable by generalized quadratures over the functional differential field $K$ is defined by the following data:

List of basic functions: all functions from the field $K$. 
List of admissible operations: Operation of composition with a function $\phi$ representable by generalized quadratures that takes $f$ to $\phi \circ f$, Arithmetic operations, Differentiation, Integration, Operation of taking exponential of integral, Operation of solving algebraic equations.

Theorem 8. A (possibly multivalued) function on the Riemann surface $U$ belongs to the class of generalized quadratures over a functional differential field $K$ if and only if it belongs to some extension of $K$ by generalized quadratures.

Theorem 8 follows from Lemma 6 (all needed arguments can be found in [3]).

5. Generalized Riccati's equation. In this section we define the generalized Riccati's equation and reduce Theorem 1 to Theorem 4. In this section we also generalize Theorem 1 for nonlinear homogeneous equations (this generalization will not be used in the next sections).

Assume that $u$ is the logarithmical derivative of a non identically equal to zero meromorphic function $y$, i.e the relation $y^{\prime}=u y$ holds.

Definition 6. Let $D_{n}$ be a polynomial in $u$ and in its derivatives $u, u^{\prime}, \ldots, u^{(n-1)}$ up to order $(n-1)$ defined by induction by the following conditions:

$$
D_{0}=1 ; \quad D_{k+1}=\frac{d D_{k}}{d x}+u D_{k}
$$

Lemma 9. 1) The polynomial $D_{n}$ has integral coefficients and $\operatorname{deg} D_{n}=n$. The degree $n$ homogeneous part of $D_{n}$ equals to $u^{n}$ (i.e. $D_{n}=u^{n}+\tilde{D}_{n}$ where $\operatorname{deg} \tilde{D}_{n}<$ $n$ ). 2) If $y$ is a function whose logarithmic derivative equals to $u$ (i.e. if $y^{\prime}=u y$ ) then for any $n \geq 0$ the relation $y^{(n)}=D_{n}(u) y$ holds.

Both claims of Lemma 9 can be easily checked by induction.

Consider a homogeneous linear differential equation (1) whose coefficients $a_{i}$ belong to a differential field $K$.

Definition 7. The equation

$$
D_{n}+a_{1} D_{n-1}+\cdots+a_{n} D_{0}=0
$$

of order $n-1$ is called the generalized Riccati's equation for the homogeneous linear differential equation (1).

Lemma 10. A non identically equal to zero function y satisfies the linear differential equation (1) if and only if its logarithmic derivative $u=y^{\prime} / y$ satisfies the generalized Riccati's equation (4).

Proof. Let $y$ be a nonzero solution of (1) and let $u$ be its logarithmic derivative. Then dividing (1) by $y$ and using the identity $y^{(k)} / y=D_{k}(u)$ we obtain that $u$ satisfies (4). If $u$ is a solution of (4) then multiplying (4) by $y$ and using the identity $y^{(k)}=D_{k}(u) y$ we obtain that $y$ is a non zero solution of (1).

Corollary 11. 1) The equation (1) has a non zero solution representable by generalized quadratures over $K$ if and only if the equation (4) has a solution representable by generalized quadratures over $K$. 
2) The equation (1) has a solution $y$ of the form $y=\exp z$ where $z^{\prime}=f$ is an algebraic function over $K$ if and only if the equation (4) has an algebraic solution over $K$.

Proof. 1) A non zero function $y$ is representable by generalized quadratures over $K$ if and only if its logarithmic derivative $u=y^{\prime} / y$ is representable by generalized quadratures over $K$.

2) A function $y$ is equal to $\exp z$ where $z^{\prime}=f$ if and only if its logarithmic derivative is equal to $f$.

The generalized Riccati's equation (4) satisfies the conditions of Theorem 4 . Thus Theorem 1 follows from Theorem 4 and from corollary 11 .

Let us generalize the results of this section. Consider an order $n$ homogeneous equation

$$
P\left(y, y^{\prime}, \ldots, y^{(n)}\right)=0
$$

where $P$ is a degree $m$ homogeneous polynomial in $n+1$ variables $x_{0}, x_{1}, \ldots, x_{n}$ over a functional differential field $K$.

Definition 7'. The equation

$$
P\left(D_{0}, D_{1}, \ldots, D_{n}\right)=0
$$

of order $n-1$ is called the generalized Riccati's equation for the homogeneous equation (1').

Lemma 10'. A non identically equal to zero function y satisfies the homogeneous equation (1') if and only if its logarithmic derivative $u=y^{\prime} / y$ satisfies the generalized Riccati's equation (4').

Corollary 11'. 1) The equation (1') has a non zero solution representable by generalized quadratures over $K$ if and only if the equation (4') has a solution representable by generalized quadratures over $K$.

2) The equation (1') has a solution $y$ of the form $y=\exp z$ where $z^{\prime}=f$ is an algebraic function over $K$ if and only if the equation (4') has an algebraic solution over $K$.

Lemma 10' and Corollary 11' can be proved exactly in the way as Lemma 10 and Corollary 11'.

Let us defined the $\xi$-weighted degree $\operatorname{deg}_{\xi} x^{p}$ of the monomial $x^{p}=x_{0}^{p_{0}} \cdot \ldots \cdot x_{n}^{p_{n}}$ by the following formula:

$$
\operatorname{deg}_{\xi} x^{p}=\sum_{i=0}^{i=n} i m_{i}
$$

We will say that a polynomial $P\left(x_{0}, \ldots, x_{n}\right)$ satisfies the $\xi$-weighted degree condition if the sum of coefficients of all monomials in $P$ having the biggest $\xi$-weighted degree is not equal to zero. A polynomial $P$ having a unique monomial with the biggest $\xi$-weighted degree automatically satisfies this condition. For example a degree $m$ polynomial $P$ containing a term $a x_{n}^{m}$ with $a \neq 0$ automatically satisfies $\xi$-weighted degree condition. 
Theorem 1'. Consider the homogeneous equation (1') with the polynomial P satisfying the $\xi$-weighted degree condition. If this equation has a non zero solution representable by generalized quadratures over $K$ then it necessarily has a solution of the form $y_{1}=\exp z$ where $z^{\prime}$ is algebraic over $K$.

Proof. It is easy to check that if the polynomial $P$ satisfies the $\xi$-weighted degree condition then the generalized Riccati's equation (4') satisfies the conditions of Theorem 4. Thus Theorem 1' follows from Theorem 4 and corollary 11'.

Remark. There exists a complete analog of Galois theory for linear homogeneous differential equations (see [4]). Theorem 1 can be proved using this theory. The differential Galois group of a nonlinear homogeneous differential equation (1') could be very small and for such equation a complete analog of Galois theory does not exist. Thus Theorem 1' can not be proved in a similar way.

\section{Special eXtensions of FUnCtional DifFEREnTial FIELDS}

In the chapter we consider simple extensions of functional differential fields. We also consider algebraic extensions of fields of rational functions over functional fields. In the section 6 we state Theorem 13 (generalized Newton's Theorem) playing a crucial role for these notes. Section 9.3 and 10.3 contains first steps for our proof of Theorem 4 .

6. Finite extensions of fields of rational functions. In this section we will discuss finite extensions of the field $K(y)$ of rational functions over a subfield $K$ of the field of meromorphic function on a connected Riemann surface $U$.

Let $F$ be extension of $K(y)$ by a root $z$ of a degree $m$ polynomial $P(z) \in(K[y])[z]$ over the ring $K[y]$ irreducible over the field $K(y)$. Let $X$ be the product $U \times \mathbb{C}^{1}$ where $\mathbb{C}^{1}$ is the standard complex line with the coordinate function $y$. An element of the field $K(y)$ can be considered as meromorphic function on $X$. One can associate with the element $z \in F$ a multivalued algebroid function on $X$ defined by equation $P(z)=0$. Let $D(y)$ be the discriminant of the polynomial $P$. Let $\Sigma \subset U \times \mathbb{C}^{1}=X$ be the hypersurface defined by equation $p_{m}(y) \cdot D(y)=0$ where $p_{m}(y)$ is the leading coefficient of the polynomial $P$.

Lemma 12. 1) About a point $x \in X \backslash \Sigma$ the equation $P(z)=0$ defines $m$ germs $z_{i}$ of analytic functions whose values at $x$ are simple roots of polynomial $P$. 2) Let $x$ be the point $(a, y) \in U \times \mathbb{C}^{1} \backslash \Sigma$. Then the field $F$ is isomorphic to the extension $K_{a}\left(y, z_{i}\right)$ of the field $K_{a}$ of germs at $a \in U$ of functions from the field $K$ (considered as germs at $x=(a, y)$ of functions independent of $y)$ extended by the independent variable $y$ and by the germ $z_{i}$ at $x$ satisfying the equation $P(z)=0$.

Proof. The statement 1) follows from the implicit function theorem. The statement 2) follows from 1).

Below we state Theorem 13 which is a generalization of Newton's Theorem about expansion of an algebraic functions into converging Puiseux series. It is stated without proof (see comments in the introduction).

We use notations introduced in the beginning of this section. Let $z_{i}$ be an element satisfying a polynomial equation $P(z)=0$ over the ring $K[y]$, where $K$ is a subfield of the field of meromorphic functions on $U$. Then there exists a finite extension $K_{P}$ of the field $K$ associated with the polynomial $P$ such that the following theorem holds. 
Theorem 13. There is a finite covering $\pi: U_{P} \rightarrow U \backslash O_{P}$ where $O_{P} \subset U$ is a discrete subset, such that the following properties hold:

1) the extension $K_{P}$ can be realized by a subfield of the field of meromorphic functions on $U_{P}$ containing the field $\pi^{*} K$ isomorphic to $K$.

2) there is a continuous positive function $r: U \backslash O_{P} \rightarrow \mathbb{R}$ such that in the open domain $W \subset\left(U \backslash O_{P}\right) \times \mathbb{C}^{1}$ defined by the inequality $|y|>r(a)$ all $m$ germs $z_{i}$ of $z$ at a point $(a, y)$ can be developed into converging Puiseux series

$$
z_{i}=z_{i_{k}} y^{\frac{k}{p}}+z_{i_{k-1}} y^{\frac{k-1}{p}}+\ldots
$$

whose coefficients $z_{i_{j}}$ are germs of analytic functions at the point $a \in U \backslash O_{P}$ having analytical continuation as regular functions on $U_{P}$ belonging to the field $K_{P}$.

Theorem 13 for a special case when the field $K$ is a subfield of the field of complex numbers it is natural to name the Newton's Theorem. One can consider $K$ as a field of constant functions on any connected Riemann surface $U$. One can chose $O_{P}$ to be the empty set, $U_{P}$ to be equal $U$, projection $\pi: U_{P} \rightarrow U$ to be the identity map, the function $r: U \rightarrow \mathbb{R}$ to be a big enough constant. In this case Theorem 13 states that an algebraic function $z$ has a Puiseux expansion at infinity whose coefficients belong to a finite extension $K_{P}$ of the field $K$. This statement can be proved by Newton's polygon method.

Let $F$ be an extension of $K(y)$ by a root $z$ of polynomial $P$ and let $K_{P}$ be the finite extension of the field $K$ introduced in Theorem 13. The extension $F_{P}$ of the field $K_{P}(y)$ by $z$ is easy to deal with. Denote the product $U_{P} \times \mathbb{C}^{1}$ by $X_{P}$.

Lemma 14. Let $x \in X_{P}$ be the point $\left(a, y_{0}\right) \in U_{P} \times \mathbb{C}^{1}$. Then the field $F_{P}$ is isomorphic to the extension $K_{P, a}\left(y, z_{i}\right)$ of the field $K_{P, a}$ of germs at $a \in U_{P}$ of functions from the field $K_{P}$ (considered as germs at $x=\left(a, y_{0}\right)$ of functions independent of $y$ ) extended by the independent variable $y$ and by the germ at $x$ of the function $z_{i}$ defined by (5).

Lemma 14 follows from Theorem 13.

7. Finite extensions of differential fields. In this section we discuss finite extensions of functional differential fields.

Let

$$
P(z)=z^{n}+a_{1} z^{n-1}+\cdots+a_{n}
$$

be an irreducible polynomial over $K, P \in K[z]$. Suppose that a functional differential field $F$ contains $K$ and a root $z$ of $P$.

Lemma 15. The field $K(z)$ is stable under the differentiation.

Proof. Since $P$ is irreducible over $K$, the polynomial $\frac{\partial P}{\partial z}$ has no common roots with $P$ and is different from zero in the field $K[z] /(P)$. Let $M$ be a polynomial satisfying a congruence $M \frac{\partial P}{\partial z} \equiv-\frac{\partial P}{\partial x}(\bmod P)$. Differentiating the identity $P(z)=0$ in the field $F$, we obtain that $\frac{\partial P}{\partial z}(z) z^{\prime}+\frac{\partial P}{\partial x}(z)=0$, which implies that $z^{\prime}=M(z)$. Thus the derivative of the element $z$ coincides with the value at $z$ of the polynomial $M$. Lemma 15 follows from this fact.

Let $K \subset F$ and $\hat{K} \subset \hat{F}$ be functional differential fields, and $P, \hat{P}$ irreducible polynomials over $K, \hat{K}$ correspondingly. Suppose that $F, \hat{F}$ contain roots $z, \hat{z}$ of $P, \hat{P}$. 
Theorem 16. Assume that there is an isomorphism $\tau: K \rightarrow \hat{K}$ of differential fields $K, \hat{K}$ which maps coefficients of the polynomial $P$ to the corresponding coefficients of the polynomial $\hat{P}$. Then $\tau$ can be extended in a unique way to the differential isomorphism $\rho: K(z) \rightarrow \hat{K}(\hat{z})$.

Proof of Theorem 16 could be obtain by the arguments used in the proof of Lemma 15.

8. Extension by one transcendental element. Let $U$ be a connected Riemann surface and let $K$ be a differential field of meromorphic functions on $U$. Let $\mathbb{C}^{1}$ be the standard complex line with the coordinate function $y$. Elements of the field $K(y)$ of rational functions over $K$ could be considered as meromorphic functions on $X=U \times \mathbb{C}^{1}$.

In the field $K(y)$ there are two natural operations of differentiations. The first operation $R(y) \rightarrow \frac{\partial R}{\partial x}(y)$ is defined as follows: the derivative $\frac{\partial}{\partial x}$ of the independent variable $y$ is equal to zero, and derivative $\frac{\partial}{\partial x}$ of an element $a \in K$ is equal to its derivative $a^{\prime}$ in the field $K$. For the second operation $R(y) \rightarrow \frac{\partial R}{\partial y}(y)$ the derivative of an element $a \in K$ is equal to zero and the derivative of the independent variable $y$ is equal to one.

Let $K \subset F$ be differential fields and let $\theta \in F$ be a transcendental element over $K$. Assume that $\theta^{\prime} \in K\langle\theta\rangle$. Under this assumption the field $K\langle\theta\rangle$ has a following description.

Lemma 17. 1) The map $\tau: K\langle\theta\rangle \rightarrow K(y)$ such that $\tau(\theta)=y$ and $\tau(a)=a$ for $a \in K$ provides an isomorphism between the field $K\langle\theta\rangle$ considered without the operation of differentiation and the field $K(y)$ of rational functions over $K$. 2) If $\tau\left(\theta^{\prime}\right)=w \in K(y)$ then for any $R \in K(y)$ and $z \in K\langle\theta\rangle$ such that $\tau(z)=R$ the following identity holds

$$
\tau\left(z^{\prime}\right)=\frac{\partial R}{\partial x}+\frac{\partial R}{\partial y} w
$$

Proof. The first claim of the lemma is straightforward. The second claim follows from the chain rule.

Let $\Theta \subset X=U \times \mathbb{C}^{1}$ be the graph of function $\theta: U \rightarrow \mathbb{C}^{1}$. The following lemma is straightforward.

Lemma 18. The differential field $K\langle\theta\rangle$ is isomorphic to the field $\left.K(y)\right|_{\Theta}$ obtained by restriction on $\Theta$ of functions from the field $K(y)$ equipped with the differentiation given by (7). For any point $a \in \Theta$ The differential field $K\langle\theta\rangle$ is isomorphic to the differential field of germs at $a \in \Theta$ of functions from $\left.K(y)\right|_{\Theta}$.

9. An extension by integral. In this section we consider extensions of transcendental degree one of a differential field $K$ containing an integral $y$ over $K$ which does not belong to $K, y \notin K$.

9.1. A pure transcendental extension by integral. Let $\theta$ be an integral over $K$,i.e $\theta^{\prime}=f \in K$. Assume that $\theta$ is a transcendental element over $K{ }^{4}$

\footnotetext{
${ }^{4}$ Easy to check that if an integral $\theta$ over $K$ does not belong to $K$, then $\theta$ is a transcendental element over $K$ (see [3]). We will not use this fact.
} 
Lemma 19. 1) The field $K\langle\theta\rangle$ is isomorphic to the field $K(y)$ of rational functions over $K$ equipped with the following differentiation

$$
R^{\prime}=\frac{\partial R}{\partial x}+\frac{\partial R}{\partial y} f
$$

2) For every complex number $\rho \in \mathbb{C}$ the map $\theta \rightarrow \theta+\rho$ can be extended to the unique isomorphism $G_{\rho}: K\langle\theta\rangle \rightarrow K\langle\theta\rangle$ which fixes elements of the field $K$.

3) Each isomorphism of $K\langle\theta\rangle$ over $K$ is an isomorphism $G_{\rho}$ for some $\rho \in \mathbb{C}$. Thus the Galois group of $K\langle\theta\rangle$ over $K$ is the additive group of complex numbers $\mathbb{C}$.

Proof. The claim 1) follows from Lemma 17. For any $\rho \in \mathbb{C}$ the element $\theta_{\rho}=\theta+\rho$ is a transcendental element over $K$ and $\theta_{\rho}^{\prime}$ equals to $f$. Thus the claims 2 ) is correct. The claim 3) followst from 2) because if $y^{\prime}=f$ then $y=\theta_{\rho}$ for some $\rho \in \mathbb{C}$.

9.2. A generalized extension by integral. According to Lemma 19 the differential field $K\langle\theta\rangle$ is isomorphic to the field $K(y)$ with the differentiation given by (8). Let $F$ be an extension of $K\langle\theta\rangle$ by an element $z \in F$ which satisfies some equation $\tilde{P}(z)=0$ where $\tilde{P}$ is an irreducible polynomial over $K\langle\theta\rangle$. The isomorphism between $K\langle\theta\rangle$ and $K(y)$ transforms the polynomial $\tilde{P}$ into some polynomial $P$ over $K(y)$. Below we use notation from section 6 and deal we the multivalued algebroid function $z$ on $X$ defined by $P(z)=0$.

Assume that at a point $x \in X$ there are germs of analytic functions $z_{i}$ satisfying the equation $P\left(z_{i}\right)=0$. Let $\theta_{\rho}$ be the function $(\theta+\rho): U_{P} \rightarrow \mathbb{C}^{1}$ and let $\Theta_{\rho} \subset$ $X=U \times \mathbb{C}^{1}$ be its graph. The point $x=(p, q) \in U \times \mathbb{C}^{1}$ belongs to the graph $\Theta_{\rho(x)}$ for $\rho(x)=q-\theta(p)$.

Let $\left.K(y)\right|_{\Theta_{\rho(x)}}$ be the differential field of germs at the point $x \in \Theta_{\rho(x)}$ of restrictions on $\Theta_{\rho(x)}$ of functions from the field $K(y)$ equipped with the differentiation given by $(8)$.

Lemma 20. The differential field $F$ is isomorphic to the finite extension of the differential field $\left.K(y)\right|_{\Theta_{\rho(x)}}$ obtained by adjoining the germ at $x \in \Theta_{\rho(x)}$ of the restriction to $\Theta_{\rho(x)}$ of an analytic germ $z_{i}$ satisfying $P\left(z_{i}\right)=0$.

Proof. For the trivial extension $F=K\langle\theta\rangle$ Lemma 20 follows from Lemmas 18, 19 . Theorem 16 allows to complete the proof for non trivial finite extensions $F$ of $K\langle\theta\rangle$.

According to section 6 with the polynomial $P$ over $K(y)$ one can associate the finite extension $K_{P}$ of the field $K$ and the Riemann surface $U_{P}$ such that Theorem 13 holds. Since $K$ is functional differential field the field $K_{P}$ has a natural structure of functional differential field. Below we will apply Lemma 20 taking instead of $K$ the field $K_{P}$ and considering the extension $F_{P} \supset K_{P}\langle\theta\rangle$ by the same algebraic element $z \in F$. The use of $K_{P}$ instead of $K$ allows to apply the expansion (5) for $z_{i}$.

Theorem 21. Let $x \in X_{P}=U_{P} \times \mathbb{C}^{1}$ be a point $\left(a, y_{0}\right)$ with $|y|>>0$. The differential field $F_{P}$ is isomorphic to the extension of the differential field of germs at the point $a \in U_{P}$ of functions from the differential fiels $K_{P}$ by the following germs: by the germ at a of the integral $\theta_{\rho(x)}$ of the function $f \in K$, and by a germ at a of the composition $z_{i}\left(\theta_{\rho}\right)$ where $z_{i}$ is a germ at $x$ of a function given by a Puiseux series (5).

Proof. Theorem 21 follow from Lemma 20 and Theorem 13. 
9.3. Solutions of equations in a generalized extension by integral. We will use notations from sec. 9.1 and 9.2.

Let $T\left(u, u^{\prime}, \ldots, u^{(n)}\right.$ by a polynomial in independent function $u$ and its derivatives with coefficients from the functional differential field $K$. Consider the equation

$$
T\left(u, u^{\prime}, \ldots, u^{(N)}\right)=0 .
$$

In general the derivative of the highest order $u^{(N)}$ cannot be represented as a function of other derivatives via the relation (9). Thus even existence of local solutions of (9) is problematic and we have no information about global behavior of its solutions.

Assume that (9) has a solution $z$ in a generalized extension by integral $F \supset K\langle\theta\rangle$ of $K$. The solution $z$ has a nice global property: it is a meromorphic function on a Riemann surface $U_{P}$ with a projection $\pi: U_{P} \rightarrow U$ which proves a locally trivial covering above $U \backslash O_{P}$, where $O_{P} \subset U$ is discrete subset.

Moreover existence of a solution $z$ implies existence of a family $z(\rho)$ of similar solutions depending on a parameter $\rho$ : one obtains such family of solutions by using an integral $\theta+\rho$ instead of the integral $\theta$ (see Lemma 20). If the parameter $\rho$ has big absolute value $|\rho|>>0$ for a point $a \in U_{P}$ of the germ $z(\rho)$ can be expand in the Piueux series in $\theta_{\rho}$ :

$$
z_{i}(\rho)=z_{i_{k}} \theta_{\rho}^{\frac{k}{p}}+z_{i_{k-1}} \theta_{\rho}^{\frac{k-1}{p}}+\ldots
$$

The series is converging and so it can be differentiate using the relation $\theta_{\rho}^{\prime}=f$.

Lemma 22. If $z_{i_{k}}^{\prime} \neq 0$ then the leading term of the Puiseux series for $z_{i}(\rho)^{\prime}$ is $z_{i_{k}}^{\prime} \theta_{\rho}^{\frac{k}{p}}$. Otherwise the leading term has degree $<\frac{k}{p}$. The leading term of the derivative of any order of $z_{i_{k}}$ has degree $\leq \frac{k}{p}$.

Let us plug into the differential polynomial $T\left(u, u^{\prime}, \ldots, u^{(N)}\right)$ the germ (10) and develop the result into Puiseux series in $\theta_{\rho}$. If the germ $z_{i}(\rho)$ is a solution of the equation (9) then all terms of this Puiseux series are equal to zero. In particular the leading coefficient is zero. This observation is an important step for proofing Theorem 4.

10. An extension by an exponential of integral. In this section we consider extensions of transcendental degree one of a differential field $K$ containing an exponential integral $y$ over $K$ which is not algebraic over $K$.

10.1. A pure transcendental extension by an exponential integral. Let $\theta$ be an an exponential of integral over $K$, i.e $\theta^{\prime}=f \theta$ where $f \in K$. Assume that $\theta$ is a transcendental element over $K .^{5}$

Lemma 23. 1) The field $K\langle\theta\rangle$ is isomorphic to the field $K(y)$ of rational functions over $K$ equipped with the following differentiation

$$
R^{\prime}=\frac{\partial R}{\partial x}+\frac{\partial R}{\partial y} f y
$$

\footnotetext{
${ }^{5}$ Easy to check that if an an exponential of integral $\theta$ over $K$ is algebraic over $K$, then $\theta$ is a radical over $K$, i.e. $\theta^{k} \in K$ for some positive integral $k$ (see [3]). We will not use this fact.
} 
2) For every complex number $\mu \in \mathbb{C}^{*}$ not equal to zero the map $\theta \rightarrow \mu \theta$ can be extended to the unique isomorphism $G_{\mu}: K\langle\theta\rangle \rightarrow K\langle\theta\rangle$ which fixes elements of the field $K$.

3) Each isomorphism of $K\langle\theta\rangle$ over $K$ is an isomorphism $G_{\mu}$ for some $\mu \in$ $\mathbb{C}^{*}$. Thus the Galois group of $K\langle\theta\rangle$ over $K$ is the multiplicative group of complex numbers $\mathbb{C}^{*}$.

Proof. The claim 1) follows from Lemma 17. For any $\mu \in \mathbb{C}^{*}$ the element $\theta_{\mu}=\mu \theta$ is a transcendental element over $K$ and $\theta_{\mu}^{\prime}=f \theta$. Thus the claims 2) is correct. The claim 3) follows from 2) because if $y^{\prime}=f y$ and $y \neq 0$ then $y=\theta_{\mu}$ for some $\mu \in \mathbb{C}^{*}$.

10.2. A generalized extension by exponential of integral. According to Lemma 23 the differential field $K\langle\theta\rangle$ is isomorphic to the field $K(y)$ with the differentiation given by (11). Let $F$ be an extension of $K\langle\theta\rangle$ by an element $z \in F$ which satisfies some equation $\tilde{P}(z)=0$ where $\tilde{P}$ is an irreducible polynomial over $K\langle\theta\rangle$. The isomorphism between $K\langle\theta\rangle$ and $K(y)$ transforms the polynomial $\tilde{P}$ into some polynomial $P$ over $K(y)$. Below we use notation from section 6 and deal we the multivalued algebroid function $z$ on $X$ defined by $P(z)=0$.

Assume that at a point $x \in X$ there are germs of analytic function $z_{i}$ satisfying the equation $P\left(z_{i}\right)=0$. Let $\theta_{\mu}$ be the function $(\mu \theta): U_{P} \rightarrow \mathbb{C}^{1}$ and let $\Theta_{\mu} \subset X=$ $U \times \mathbb{C}^{1}$ be its graph. The point $x=(p, q) \in U \times \mathbb{C}^{1}$ where $q \neq 0$ belongs to the graph $\Theta_{\mu(x)}$ for $\mu(x)=q \cdot \theta(p)^{-1}$.

Let $\left.K(y)\right|_{\Theta_{\mu(x)}}$ be the differential field of germs at the point $x \in \Theta_{\mu(x)}$ of restrictions on $\Theta_{\mu(x)}$ of functions from the field $K(y)$ equipped with the differentiation given by (11).

Lemma 24. The differential field $F$ is isomorphic to the finite extension of the differential field $\left.K(y)\right|_{\Theta_{\mu(x)}}$ obtained by adjoining the germ at $x \in \Theta_{\mu(x)}$ of the restriction to $\Theta_{\mu(x)}$ of an analytic germ $z_{i}$ satisfying $P\left(z_{i}\right)=0$.

Proof. For the trivial extension $F=K\langle\theta\rangle$ Lemma 24 follows from Lemmas 18, 23 . Theorem 16 allows to complete the proof for non trivial finite extensions $F$ of $K\langle\theta\rangle$.

According to section 6 with the polynomial $P$ over $K(y)$ one can associate the finite extension $K_{P}$ of the field $K$ and the Riemann surface $U_{P}$ such that Theorem 13 holds. Since $K$ is functional differential field the field $K_{P}$ has a natural structure of functional differential field. Below we will apply Lemma 24 taking instead of $K$ the field $K_{P}$ and considering the extension $F_{P} \supset K_{P}\langle\theta\rangle$ by the same algebraic element $z \in F$. The use of $K_{P}$ instead of $K$ allows to apply the expansion (5) for $z_{i}$.

Theorem 25. Let $x \in X_{P}=U_{P} \times \mathbb{C}^{1}$ be a point $\left(a, y_{0}\right)$ with $|y|>>0$. The differential field $F_{P}$ is isomorphic to the extension of the differential field of germs at the point $a \in U_{P}$ exponential of integral $\theta_{\mu(x)}$, where $\theta_{\mu(x)}^{\prime}=f \theta_{\mu(x)}$ for the function $f \in K$, and by a germ at a of the composition $z_{i}\left(\theta_{\mu}\right)$ where $z_{i}$ is a germ at $x$ of a function given by a Puiseux series (5).

Proof. Theorem 25 follow from Lemma 24 and Theorem 13.

10.3. Solutions of equations in a generalized extension by exponential of integral. Assume that (9) has a solution $z$ in a generalized extension by exponential of integral $F \supset K\langle\theta\rangle$ of $K$. Solution $z$ has a nice global property: it is 
a meromorphic function on a Riemann surface $U_{P}$ with a projection $\pi: U_{P} \rightarrow U$ which proves a locally trivial covering above $U \backslash O_{P}$, where $O_{P} \subset U$ is discrete subset.

Moreover existence of a solution $z$ implies existence of family $z(\mu)$ of similar solutions depending on a parameter $\mu \in \mathbb{C}^{*}$ : one obtains such family of solutions by using an exponential of integral $\mu \theta$ instead of the exponential of integral $\theta$ (see Lemma 24). If the parameter $\mu$ has big absolute value $\mu \mid>>0$ for a point $a \in U_{P}$ of the germ $z(\mu)$ can be expand in the Piueux series in $\theta_{\mu}$ :

$$
z_{i}(\mu)=z_{i_{k}} \theta_{\mu}^{\frac{k}{p}}+z_{i_{k-1}} \theta_{\mu}^{\frac{k-1}{p}}+\ldots
$$

The series is converging and so it can be differentiate using the relation $\theta_{\mu}^{\prime}=f \theta_{\mu}$.

Lemma 26. If $z_{i_{k}}^{\prime}+\frac{k}{p} z_{i_{k}} \neq 0$ then the leading term of the Piueux series for $z_{i}(\mu)^{\prime}$ is $\left(z_{i_{k}}^{\prime}+\frac{k}{p} z_{i_{k}}\right) \theta_{\mu}^{\frac{k}{p}}$. Otherwise the leading term has degree $<\frac{k}{p}$. The leading term of derivative of any order of $z_{i_{k}}$ has degree $\leq \frac{k}{p}$.

Let us plug into the differential polynomial $T\left(u, u^{\prime}, \ldots, u^{(N)}\right)$ the germ (12) and develop the result into Puiseux series in $\theta_{\mu}$. If the germ $z_{i}(\mu)$ is a solution of the equation (9) then all terms of this Piueux series are equal to zero. In particular the leading coefficient is zero. This observation is an important step for proofing Theorem 4.

\section{Proof of Rosenlicht's Theorem}

Here we complete an elementary proof of Theorem 4 discovered by Maxwell Rozenlicht [2]. We will proof first the simplier theorems 27, 28 of a similar nature.

Theorem 27. Assume that the equation (2) over a functional differential field $K$ has a solution $z \in F$ where $F$ is a generalized extension by integral of $K$. Then (2) has a solution in the algebraic extension $K_{P}$ of $K$ associated with the polynomial $P$.

Proof. If the constant term of the differential polynomial $T\left(u, u^{\prime}, u^{\prime \prime}, \ldots\right)=u^{n}-$ $Q\left(u, u^{\prime}, u^{\prime \prime}, \ldots\right)$ is equal to zero, then (2) has solution $u \equiv 0$ belonging to $K$. In this case we have nothing to prove.

Below we will assume that the constant term $T_{0}$ of $T$ is not equal to zero. Thus the differential polynomial $T$ has two special terms: the term $u^{n}$ which is the only term of highest degree $n$ and the term $T_{0}$ which is the only term of smallest degree zero.

Assume that (2) has a solution $z$ in a generalized extension by integral $F \supset K\langle\theta\rangle$ of $K$. According to sec. 9.3 the existence of such a solution $z$ implies the existence of family $z(\rho)$ of germs of solutions depending on a parameter $\rho$ such that when the whose absolute value $\rho \mid$ is big enough $z(\rho)$ can be expanded in Puiseux series (10) in $\theta_{\rho}$.

We will show that the degree $\frac{k}{p}$ of the leading term in (10) is equal to zero and the leading coefficient $z_{i_{0}} \in K_{P}$ satisfies (2). This will proof Theorem 27 .

According to Lemma 22 the leading term of the derivative of any order of $z_{i_{k}}$ has degree $\leq \frac{k}{p}$. Thus the leading term of Puiseux series obtained by plugging (10) 
instead of $u$ into differential polynomial $Q$ has degree $<n \frac{k}{p}$. The leading term of the Puiseux series obtained by arising (10) to the $n$-th power is equal to $n \frac{k}{p}$. If $\frac{k}{p}>0$ this term can not be canceled after plugging (10) instead of $u$ into differential polynomial $T$. Thus the degree $\frac{k}{p}$ can not be positive.

Let us plug (10) into the differential polynomial $T\left(u, u^{\prime}, \ldots\right)-T_{0}$. We will obtain a Puiseux series of negative degree if $\frac{k}{p}<0$. Thus the term $T_{0}$ in the sum $\left(T-T_{0}\right) I+T_{0}$ can not be canceled. Thus $\frac{k}{p}$ can not be negative.

We proved that $\frac{k}{p}=0$. If in this case we plug (10) into the differential polynomial $T\left(u, u^{\prime}, \ldots\right)$ we obtain a Puiseux series having only one term of nonnegative degree which is equal to zero. From Lemma 22 it is easy to see that this term equals to $T\left(z_{i_{0}}, z_{i_{0}}^{\prime}, \ldots\right) \theta_{\rho}^{0}$. Thus $z_{i_{0}} \in K_{P}$ is a solution of (2). Theorem 27 is proved.

Theorem 28. Assume that equation (2) over a functional differential field $K$ has a solution $z \in F$ where $F$ is a generalized extension by an exponential of integral of $K$. Then (2) has a solution in the algebraic extension $K_{P}$ of $K$ associated with the element $z \in F$.

Proof. Theorem 28 can be proved exactly in the same way as Theorem 27 . Just instead of Lemma 22 one has to use Lemma 26. In the case when leading term of the Puiseux expansion of $z_{\mu}$ has degree zero, the leading coefficient of its derivative equals to $z_{i_{0}}^{\prime}$ (see Lemma 26). That is why the case $\frac{k}{p}=0$ in Theorem 28 can be treated exactly in the same way as in Theorem 27.

Now we ready to prove Theorem 4.

Proof of Theorem 4. By assumption the equation (2) has a solution $z \in F$ where $F$ is an extension of $K$ by generalized quadratures. By Lemma 5 there is a chain $K=F_{0} \subset \cdots \subset F_{m}$ such that $F \subset F_{m}$ and for every $i=0, \ldots, m-1$ or $F_{i+1}$ is a finite extension of $F_{i}$, or $F_{i+1}$ is a generalized extension by integral of $F_{i}$, or $F_{i+1}$ is a generalized extension by exponential integral of $F_{i}$. We prove Theorem 4 by induction in the length $m$ of the chain of extension. For $m=1$ Theorem 4 follows from Theorem 27, or from Theorem 28. Assume that Theorem 4 is true for $m=k$. A chain $F_{0} \subset F_{1} \subset \cdots \subset F_{k+1}$ provides the chain $F_{1} \subset \cdots \subset F_{k+1}$ of extensions of length $k$ for the field $F_{1}$. Thus (2) has an algebraic solution $z$ over the field $F_{1}$. The extension $F_{0} \subset \tilde{F}_{1}$, where $\tilde{F}_{1}$ is the extension of $F_{1}$ by the element $z$, is either an algebraic extension, or extension by generalized integral or extension by generalized exponential of integral. Thus for the extension $F_{0} \subset \tilde{F}_{1}$ Theorem 4 holds. We completed the inductive proof of Theorem 4.

\section{REFERENCES}

1. J. F. Ritt, Integration in finite terms, Columbia Univ. Press, New York, 1948..

2. M. Rosenlicht, An analogue of l'Hospital's rule, Proc. Amer. Math. Soc. 37 (1973), no. 2, 369-373.

3. A.G. Khovanskii, Topological Galois theory. Solvability and nonsolvability of equations in finite terms, Springer.

4. M. van der Put, M. Singer, Galois theory of linear differential equations, Grundlehren der Mathematischen Wissenschaften [Fundamental Principles of Mathematical Sciences] Vol. 328, Berlin, New York: Springer-Verlag, 2003. 